\title{
Cochlear Implant Surgery
}

\author{
Hakan Soken, Sarah E. Mowry and Marlan R. Hansen \\ Department of Otolaryngology, University of Iowa Hospitals and Clinics, Iowa City, Iowa
}

USA

\section{Introduction}

Over the past quarter of a century, cochlear implants (CIs) have become recognized as highly successful auditory rehabilitation devices for individuals with severe to profound hearing impairment. There is ample evidence of the success of electrical stimulation of the inner ear as a treatment for profound deafness.

However, the majority of persons with hearing loss are not profoundly deaf and they have some remaining usable hearing. Encouraged by promising results in traditional cochlear implant patients and by improvements in the electrode design and the signal processing, investigators have expanded the indications for implantation. Now in addition to completely deaf patients, selected subjects with residual hearing are eligible for cochlear implantation with modified electrodes. The approach involves preserving existing residual acoustic hearing (low-frequency) in an ear to be implanted. Electrical stimulation is provided via a modified $\mathrm{CI}$ for the missing high frequencies to improve speech understanding via combined acoustic and electric $(\mathrm{A}+\mathrm{E})$ hearing. The prerequisite for this form of combined stimulation (ipsilateral $A+E$ ) is a sufficient degree of residual low frequency hearing in the implanted ear. Most patients with the modified electrodes are able to achieve improved sound perception and word understanding while preserving their residual acoustic hearing (Ching et al., 1998; Hogan \& Turner, 1998). The listening condition using only A+E hearing and no hearing aid in the contralateral ear is referred to as "hybrid mode". However, these implant recipients benefit from using a hearing aid in their contralateral ear, a listening condition referred to as "combined mode". This listening situation has the potential to improve speech recognition in both quiet and noise (Gstoettner et al., 2006; Turner \& Cummings, 1999).

Since Gantz and von Ilberg first discussed the possibility of using A+ E stimulation simultaneously in patients with significant residual hearing, the concept of combined electric and acoustic stimulation has provided a focus of interest and research (von Ilberg et al., 1999; Gantz \& Turner, 2003). The Iowa Hybrid project stemmed from work by Shepherd and colleagues that showed that preservation of the apical regions of the feline cochlea could be spared anatomically and functionally following limited electrode insertion (Ni et al., 1992; Xu et al., 1997). Developments in technology and "soft" surgery techniques, combined with a better understanding of the structure and function of the inner ear allowed the first patient to be implanted with a modified electrode in 1999 (Gantz \& Turner, 2003). Work by groups in Iowa and Frankfurt have targeted hearing-impaired 
patients who are not traditionally considered CI candidates (Gantz \& Turner, 2003; Cohen et al., 2002). These patients are characterized by severe and profound thresholds at frequencies $\geq 1000 \mathrm{~Hz}$, with near-normal or mild hearing losses in the low frequencies. These patients commonly present with monosyllabic word recognition scores $<50 \%$. In these cases the aim is to preserve functional low frequency hearing while providing additional high frequency information via the $\mathrm{CI}$.

Successful implantation of this group of hearing impaired patients requires meticulous microsurgical techniques. This chapter will explore the indications for use of $\mathrm{A}+\mathrm{E}$ stimulation, patient outcomes, microsurgical techniques, electrode design and possibilities for future interventions.

\section{Indications for combined acoustic and electric stimulation}

Cochlear implantation is traditionally offered to individuals who receive limited hearing benefit from well-fit hearing aids (HAs). The definition of "limited benefit" for patients has changed appreciably in the past 15 years. The audiologic selection criteria have been expanded for both adults and pediatric patients. For adults, selection criteria have changed from a profound hearing loss and limited open-set speech recognition in the early 1990s to a $70 \mathrm{~dB}$ hearing loss and up to $50 \%$ open-set sentence speech perception. Although the broadening of the selection criteria to a $70 \mathrm{~dB}$ pure-tone-average hearing loss occurred in 1995 for the adult population, the criteria for children remain a pure-toneaverage of less than or equal to $90 \mathrm{~dB}$ hearing level. As cochlear implant technology progressed and documented outcomes exceeded early expectations, the audiologic boundaries of candidacy broadened to include patients with more residual hearing (Kiefer et al., 1998; Klenzner et al., 1999).

Severe to profound hearing impaired individuals typically derive substantial benefit from a $\mathrm{CI}$ for speech understanding and quality of life. Conventional CI users may use a HA in the contralateral ear (bimodal condition) if sufficient residual hearing is present. This listening condition has the potential to improve speech recognition, particularly in noise. Results in these patients show that bimodal listening is of significant benefit, and a strong synergistic effect of using both devices is particularly noticeable during speech testing in noise (Gantz \& Turner, 2003; Turner et al., 2004; Gstoettner et al., 2008). Ipsilateral combination of A+E hearing (hybrid listening mode) provides similar benefits for speech recognition in noise and subjective improvements in sound quality (Gstoettner et al., 2006).

Several recent studies demonstrated that acoustic amplification for hearing loss above $60 \mathrm{~dB}$ $\mathrm{HL}$ for frequencies greater than $2500 \mathrm{~Hz}$ usually provides no enhancements of speech recognition (Hogan \& Turner, 1998; Turner \& Cummings, 1999). Thus, candidates for A+E stimulation must have pure tone detection $<60 \mathrm{~dB}$ HL between $125-500 \mathrm{~Hz}$ and $<80 \mathrm{~dB} \mathrm{HL}$ above $2000 \mathrm{~Hz}$. They may have substantial word understanding scores (consonant nucleus consonant $(\mathrm{CNC})$ ) in the best aided situation between $10-60 \%$ correct in the worse hearing ear and up to $80 \%$ correct in the better hearing ear (Fig. 1). Other selection criteria include: no evidence of progressive hearing loss; no evidence of autoimmune inner ear disease; and no history of meningitis, otosclerosis or cochlear ossification. The maximum air-bone gap allowed is $15 \mathrm{~dB}$. There should also be no contraindications to use amplification devices in the implanted ear such as chronic otitis externa or a chronically draining ear. 


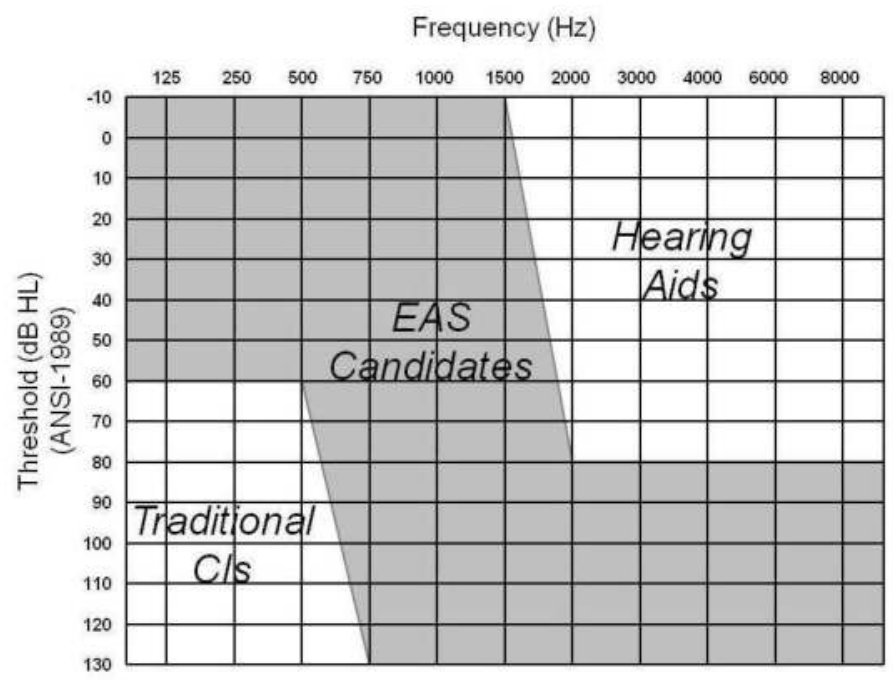

Fig. 1. Expanded audiometric selection criteria for combined electric and acoustic stimulation candidacy. HL=Hearing Level, $\mathrm{Hz}=$ Hertz.

\section{Outcomes}

In 2003, Gantz and Turner reported on 9 adults with severe high-frequency impairment received implants with either $6-\mathrm{mm}$ or $10-\mathrm{mm}$ length hybrid electrodes. Monosyllabic word understanding and consonant identification in a recorded sound-only condition were used to assess changes in speech perception. Acoustic hearing was preserved in all subjects and preoperative monosyllabic word and sentence scores were unchanged in all subjects following implantation. The scores were more than doubled using the $10-\mathrm{mm}$ implant when compared with preoperative scores achieved with hearing aids only. In the subsequent FDA trial of the Hybrid S12 10mm electrode, 87 subjects were enrolled in a larger multi-central clinical trial. Immediate hearing preservation accomplished in 85/87 (98\%) subjects. Over time (3 months to 5 years), some hearing was maintained in $91 \%$ of the group (Gantz et al., 2009).

Other modified electrodes (Cochlear Hybrid L24 and Med-El FlexEAS) are also designed to preserve hearing. Lenarz et al. (2009) recently published the preliminary data for the European trial of the L24. Of the 32 patients implanted; 24 patients were hybrid candidates and 8 patients were standard electrode candidates. Hearing was preserved within $30 \mathrm{~dB}$ of preoperative thresholds in $96 \%$ of patients and $68 \%$ were within $15 \mathrm{~dB}$. These results were stable over time. Of the 16 patients with 12 months of experience, $94 \%$ retained hearing within $30 \mathrm{~dB}$ of preoperative thresholds. Those in the European trial showed significant improvement on word scores between the 6- and 12-month post-activation marks.

As discussed previously, patients with preserved LF hearing have significant improvements in their discrimination scores following cochlear implantation. Those implanted with shorter electrode arrays, such as the Hybrid S/L or Med-El M/FLEXEAS electrodes, are achieving significant improvements in discrimination tasks as well. Patients implanted with the Hybrid S electrode continue to demonstrate improvement in CNC scores beyond 1-2 years 
after activation in the combined mode (Reiss et al., 2007). At the time of publication of the Hybrid 10 clinical trial, 68 of 87 patients in the multi-center trial had follow-up lengths of greater than 9 months. Improvements in speech reception threshold (SRT) or CNC word score occurred in $74 \%$ of patients. Nearly half of patients $(48 \%)$ had improvement in both SRT and CNC scores. Improvement on CNC testing ranged from $10 \%$ to $70 \%$ better than preoperative scores for 45 of 61 patients with long term follow-up (Gantz et al., 2009).

For those implanted with the Hybrid L24, word recognition scores improved by $21 \%$ on average; one patient demonstrated improvement from $5 \%$ to $95 \%$ on the Freiburg Monosyllabic word test (Lenarz et al., 2009).

Some patients score above $90 \%$ on the CNC monosyllabic word test in the combined mode with all electrodes. Patients implanted with the FLEXEAS electrode also scored well. Preoperative open set sentence recognition was $24 \%$ and after 12 months of use scores averaged $71 \%(\mathrm{p}<0.05)$. Monosyllable recognition also improved; preoperative scores averaged $16 \%$ on the FMS test and postoperative scores averaged $44 \%(\mathrm{p}<0.05)$. One patient in this cohort achieved scores over $90 \%$ discrimination post-operatively (Gstoettner et al., 2008).

Although cochlear implants significantly improve speech understanding in quiet, traditional CI users have difficulty in noisy environments. Distinguishing the correct words in a background of competing talkers is an even more difficult task. Normal hearing listeners are able to understand $50 \%$ of the presented words when the background noise is $30 \mathrm{~dB}$ louder; thus normal hearing listeners have a signal to noise ratio (SNR) of $-30 \mathrm{~dB}$ (lower numbers are better). For competing talkers, the average SNR in normal hearing listeners is $-15 \mathrm{~dB}$ (Turner et al., 2004). The average long electrode user requires a SNR of $+3 \mathrm{~dB}$ for unmodulated background noise and +8 for multitalker babble (MTB), meaning that the talker has to be $3 \mathrm{~dB}$ louder than competing noise or $8 \mathrm{~dB}$ louder than MTB (Nelson et al., 2003; Gantz et al., 2006).

Hybrid S recipients perform much better than traditional CI patients but not as well as normal hearing listeners in background noise. SNRs varied from -12 to $+17 \mathrm{~dB}$ in a subgroup of 27 Hybrid S patients with 12 months or greater experience. The average SNR for the Hybrid S group was -9 dB (Gantz et al., 2009). Elevated SNRs occurred in those patients who experienced $>30 \mathrm{~dB}$ changes to their LF hearing. The results for Hybrid S patients in MTB are similar to hearing impaired patients with SRTs between 81-100 dB (severe/profound).

Patients receiving the Hybrid L electrode also improved their SNR when tested in the combined mode. The average SNR preoperatively was $+12.1 \mathrm{~dB}$ and postoperatively the SNR dropped to $+2.1 \mathrm{~dB}$ (Lenarz et al., 2009).

Those with the FLEXEAS electrode also improved speech understanding in noise. Preoperative open set sentence scores in SNR of +10 were $14 \%$ and after 1 year in the EAS mode scores averaged 60\% (p<0.05) (Gstoettner et al., 2008).

Music appreciation has been a part of the research protocol for the Hybrid S/L trials. Subjects with preserved LF hearing have a distinct advantage in a number of music processing functions when compared to the traditional CI recipients. Pitch perception is one of the most basic functions of the auditory system with respect to music appreciation. Hybrid users perform better on these types of tasks when compared to long electrode users but are still significantly poorer-performing than normal hearing listeners (Gfeller et al., 
2007). When provided with lyrics to easily recognizable American songs, Hybrid users were able to identify the songs correctly $65-100 \%$ of the time, similar to normal hearing listeners. When the lyrics were removed and only the melody was presented, Hybrid patients did less well ( $50 \%$ correct) but still much better than traditional long electrode users ( $<10 \%$ correct) (Gfeller et al., 2006).

The clinical trials of $\mathrm{A}+\mathrm{E}$ patients are still in their early years; therefore the issue of longterm success rates still deserves attention. A recent study retrospectively analyzed low frequency hearing stability over time. These patients met criteria for hybrid implantation at the beginning of the study period (Yao et al., 2006). Adults demonstrated stable low frequency thresholds (changing only $1 \mathrm{~dB}$ per year over periods as long as 25 years), however for children the rate of hearing loss was generally larger and much more variable. The stability of low-frequency thresholds in actual A + E patients over long periods of time remains to be determined.

\section{Technical issues}

The goals and theories behind the development of techniques to preserve residual hearing preservation following cochlear implantation encompass more than merely inserting a standard length electrode into the cochlea under modified technique.

The loss of residual acoustic hearing during implantation is multifactorial. Electrode design, surgical technique and host responses to insertional trauma all likely contribute to postoperative hearing change and ultimately patient outcomes.

The diameter, stiffness, and length of standard intracochlear electrodes may induce substantial intracochlear damage. Histological evaluation of the cochlea after insertion of an electrode in human cadaver temporal bones demonstrated a wide range of damage to inner ear structures, including the basilar membrane, osseus spiral lamina and cochlear hair cells (Fayad et al., 1991; Eshraghi et al., 2003). Electrode position and structural damage to the cochlea can be quantified in cadaveric studies using the scale described by Eshraghi and Van De Water (2006) (Table 1). Using this scale and objective electrophysiological testing of the hearing threshold (i.e., DPOAEs and ABRs) in animal models of cochlear implant electrode trauma, experience has shown that most of the causes of postoperative hearing loss after cochlear implantation can be minimized by electrode design and optimized surgical technique (Eshraghi et al., 2003; Balkany et al., 2002).

Grade 1: No observable macroscopic trauma

Grade 2: Elevation of basilar membrane

Grade 3: Dislocation of electrode to scala vestibuli

Grade 4: Fracture of osseous spiral lamina or modiolus, or tear in tissues of stria vascularis / spiral ligament complex

Table 1. Grading system: Cochlear trauma postelectrode array implantation. (Modified from: Eshraghi et al., Comparative study of cochlear damage with three perimodiolar electrode design. Laryngoscope 2003;113:415-419) 


\subsection{Electrode features}

Taking into consideration the frequency-specific nature of the cochlea, partial insertion of standard length electrodes (Nucleus CI22M, CI24M and Nucleus 24 Contour, the Med-EL Combi $40+$ and the Advance Bionics HiFocus II) was originally considered in an effort to preserve acoustic hearing in the low tones. All standard electrode arrays are designed to be inserted to a depth exceeding 1 complete turn of the cochlea from base to apex, or $360^{\circ}$ (Fig. 2). Reports in the literature have shown the ability to preserve some residual hearing even when a standard electrode array is fully inserted into the scala tympani (Hodges et al., 1997; Balkany et al., 2006). However, passing a standard length electrode beyond the basal turn of the cochlea can result in damage to the organ of Corti due to migration of the electrode through the basilar membrane. Therefore, it is difficult to consistently maintain speech discrimination in addition to pure tones when electrodes are passed beyond the basal turn (Balkany et al., 2006).

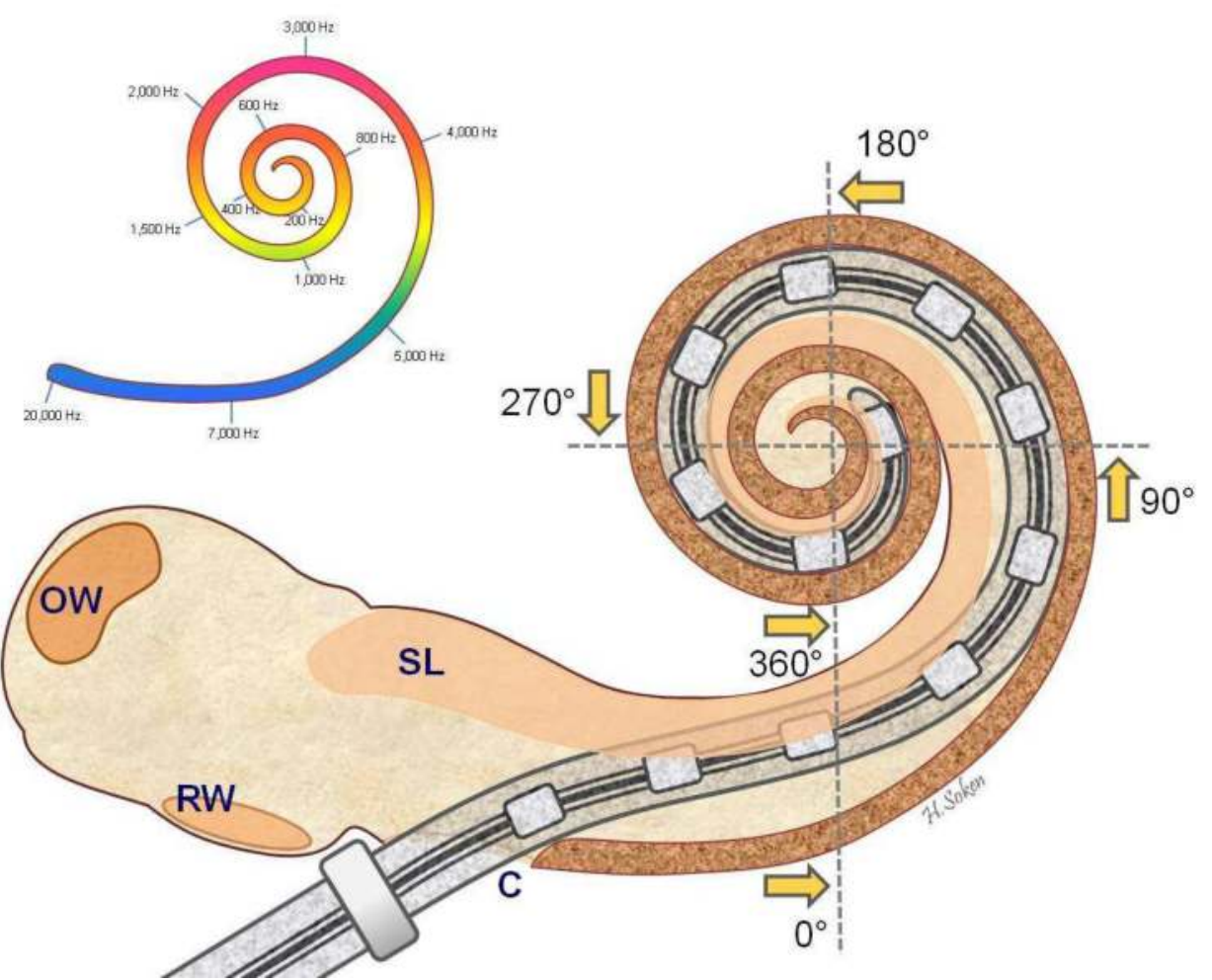

Fig. 2. Insertion depth, and frequency map of the cochlea. SL=Spiral Lamina, OW=Oval Window, RW=Round Window, C=Cochleostomy

In 1995, the University of Iowa CI research team began development of a shortened electrode array based on the Nucleus CI-24 implant in collaboration with the Cochlear Corporation. The first design, "Hybrid S8" has a reduced diameter of $6 \mathrm{~mm} \times 0.2 \mathrm{~mm} \times 0.4$ 
mm electrode with six channels. Later, the Iowa/Nucleus Hybrid (short-electrode) device was then lengthened to $10 \mathrm{~mm}$, with the electrodes placed at the distal $6 \mathrm{~mm}$, the Hybrid S12 (Fig. 3). Unique features of the Hybrid electrode include a Dacron collar to limit the intracochlear placement to $10 \mathrm{~mm}$ and a titanium marker to orient the electrode contacts toward the modiolus. The ideal insertion depth is approximately $195^{\circ}$ of the basal turn of the cochlea (Roland et al., 2008).

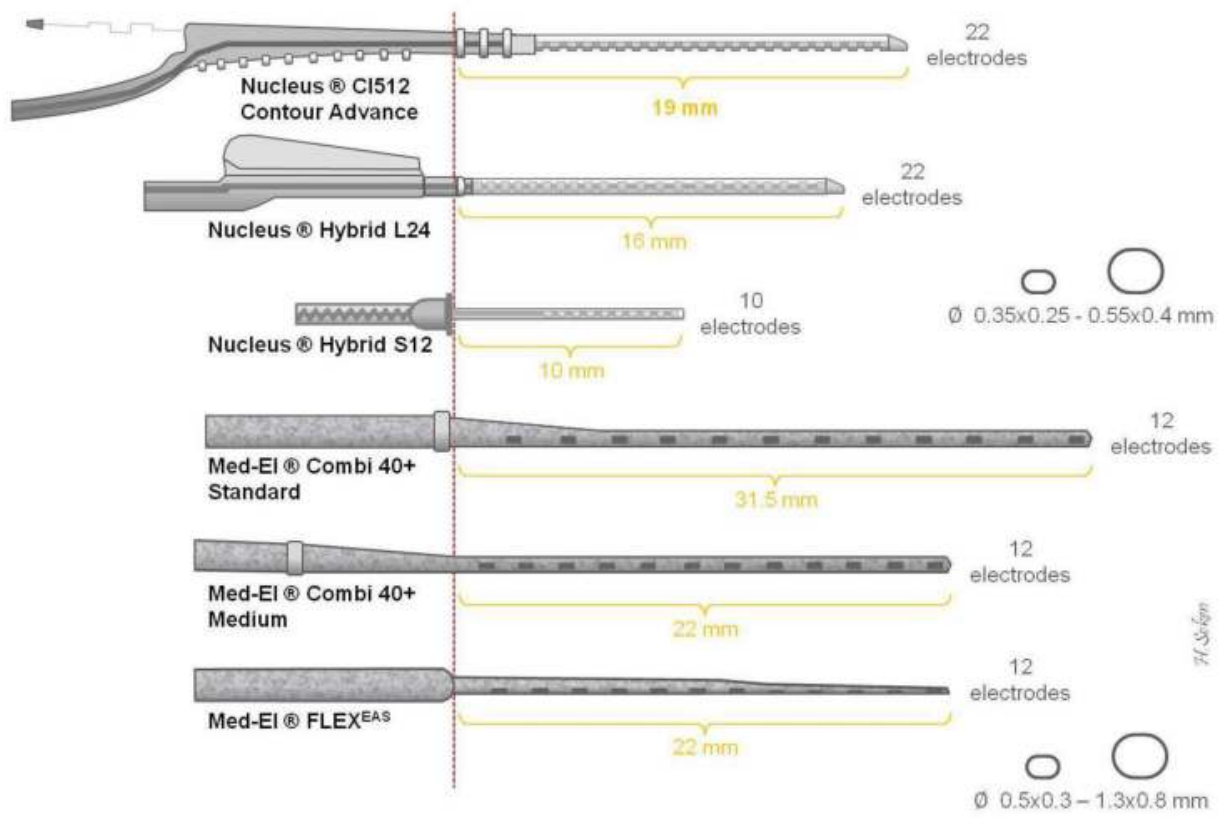

Fig. 3. Examples of various CI electrode arrays.

Another short electrode has been developed in conjunction with the Cochlear Corporation, the Hybrid L24, which is $16 \mathrm{~mm}$ length and contains 22 electrodes. The optimal insertion is through $250^{\circ}$ of the basal turn of the cochlea. This longer electrode would still preserve residual hearing in the apical portions of the cochlea, but if the low frequency hearing is lost the Hybrid L can be used as a traditional electric-processing only device as it has 22 electrodes, similar to a standard electrode (Fig. 3).

In 2004, Med-El Corporation launched a new atraumatic prototype electrode carrier FLEXEAS in an attempt to minimize the forces generated during insertion and thereby to increase the rate of residual hearing preservation to allow for $\mathrm{E}+\mathrm{A}$ hearing. Their standard length electrode, the Combi 40+, has a goal insertion length of $31 \mathrm{~mm}$. The shortened electrode, the $\mathrm{M}$, is $22 \mathrm{~mm}$ in length and the electrode includes a significantly reduced diameter of the distal portion of the electrode with a flexible tip (Fig. 3). This FLEXEAS electrode can be used for both cochleostomy and round window insertion techniques (Hochmair et al., 2006). Human temporal bone studies confirmed the intended mechanical properties for safe and atraumatic insertion (Adunka et al., 2004). 


\section{Surgical technique}

\subsection{Surgical approach}

As in all cochlear implant surgeries, an antromastoidectomy is performed, followed by a posterior tympanotomy to visualize the round window niche. The facial recess must be opened to allow complete visualization of the round window. The incudal buttress is preserved and care is taken to leave the ossicular chain intact and untouched. The bed for the receiver/stimulator is prepared before entry into the middle ear. In most temporal bones, the round window cannot be directly viewed until its bony overhang has been properly removed. The round window niche is saucerized with a $1.5 \mathrm{~mm}$ diamond burr to expose the round window membrane fully.

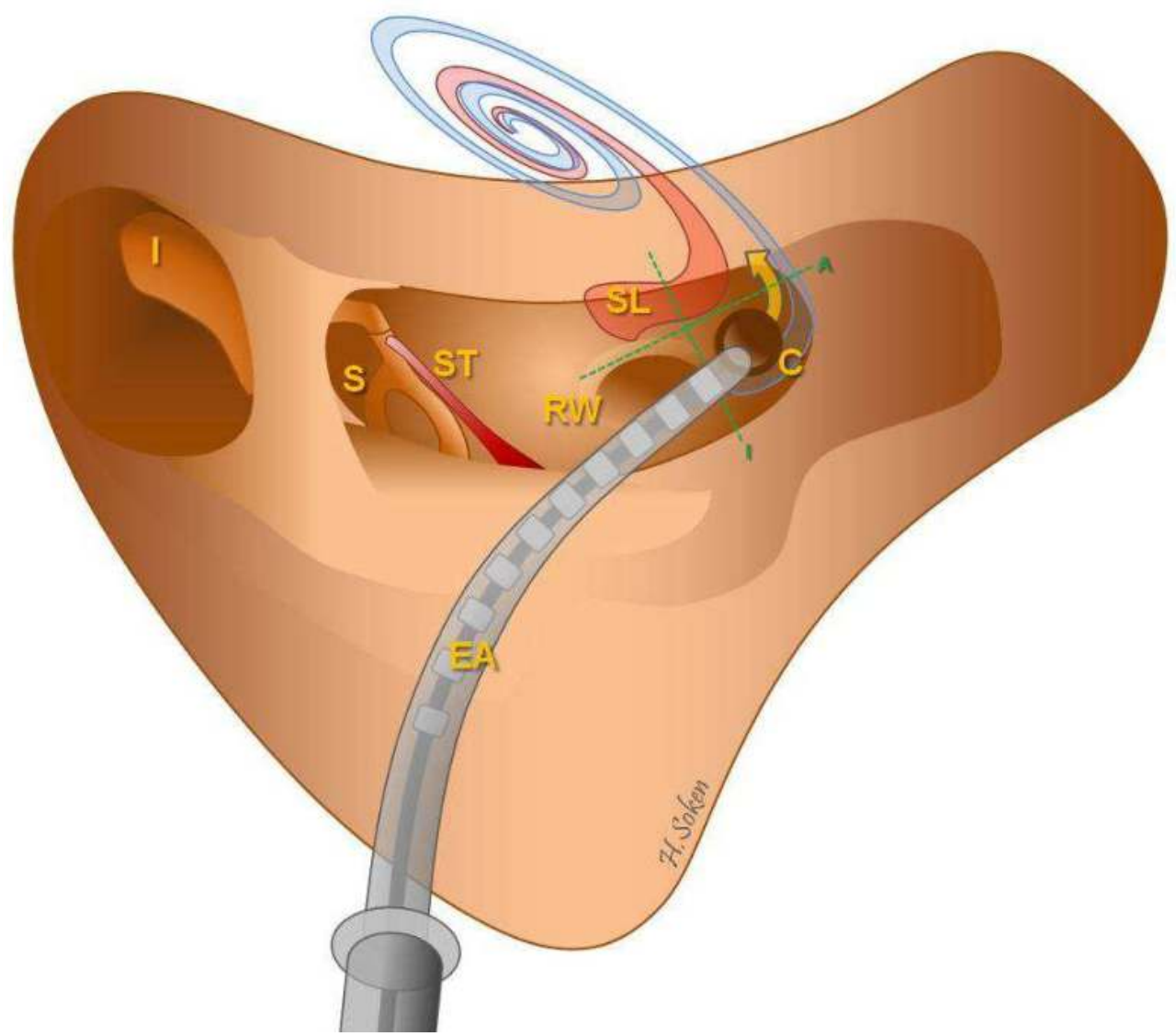

Fig. 4. Cochleostomy anteroinferior to the round window (green dotted lines) and introduction of the electrode array into the scala tympani. The electrode must be directed parallel to the posterior canal wall to ensure the electrode is directed into the basal turn of the cochlea (yellow arrow). C=Cochleostomy, EA=Electrode Array, I=Incus, RW=Round Window, S=Stapes, SL=Spiral Lamina, ST=Stapedial Tendon. 
The position and technique of creating the cochleostomy is critical to preserving residual hearing. To standardize the placement of the cochleostomy, it is placed in the anterior inferior quadrant of a box created by drawing a line at the superior margin of the round window and one that crosses perpendicular at the inferior aspect of the round window. Creation of the cochleostomy is begun in the inferior portion of the quadrant, slowly saucerizing the otic capsule bone with a $1.5 \mathrm{~mm}$ diamond bur. This position allows a "straight" path into the basal turn of the scala tympani and avoids the osseous spiral lamina when entering the scala tympani (Fig. 4). The cochleostomy hole is drilled so that the "blueline" of the endosteum will be visible. If the cochleostomy is too far superior, adjacent to the spiral ligament, a whitish color will be evident. A $0.5 \mathrm{~mm}$ diamond bur is used to penetrate the final layer of bone and the cochleostomy drilling is done at a slow speed.

Care is taken to use sufficient irrigation to avoid heating the cochlea. Contact with the bone over the facial nerve by the revolving drill shaft must be avoided, since this will heat the bone and may result in a thermal injury to the facial nerve itself. Entry into the scala tympani should not occur until bleeding is controlled and bone dust has been removed from the field.

Several other steps occur prior to cochlear entry. To assist immediate and complete sealing of the cochleostomy hole, a $1.5 \times 1.5 \mathrm{~mm}$ graft of the temporalis fascia is created and then perforated centrally. The electrode is then passed through this perforation. When implanting the Hybrid S12 it helps to secure the electrode within the mastoid. This is accomplished by drilling a pair of $1 \mathrm{~mm}$ holes in the cortical overhang of the tegmen mastoideum. A 4-0 nylon suture is then passed through these holes and the electrode is passed through the loop. The suture is then tied down laterally to secure the electrode. This suture helps to stabilize the orientation of the electrode contacts toward the modioulus and ensures that the electrode is secure within the scala tympani.

\subsection{Electrode insertion}

Immediately prior to electrode insertion, the surgeon should check that all preliminary steps have been completed. The endosteum is incised using a $0.2 \mathrm{~mm}$ right-angle hook to open the scala tympani. The diameter of the cochleostomy varies from $0.5-1.5 \mathrm{~mm}$ depending on the electrode used. Some surgeons advocate placing a trapezoid silicon sheath in the posterior tympanotomy and mastoid to guide the insertion of the electrode and to limit contamination of the electrode with blood or bone dust. To facilitate electrode insertion, the surface may be coated with surgical lubricant such as hyaluronic acid. Some surgeons place a drop of crystalline triamcinolone solution (Volon $A \circledR$ ) in the cochleostomy hole and then seal the cochleostomy with a drop of hyaluronic acid (Healon $\left.{ }^{\circledR}\right)$ to prevent the corticosteroid solution from being flushed away.

The time the cochlea remains exposed should be minimized. Once the cochlea is opened, the electrode is then inserted gently and slowly into the scala tympani. Regardless of the type of electrode, it's important that the electrode parallel the posterior canal wall to ensure the electrode is directed into the basal turn of the cochlea (Fig. 4). The tip of the electrode is guided into the cochleostomy using a smooth forceps; at no point is the electrode lead circumferentially grasped or forcefully pushed into the cochlea. To minimize intrascalar pressure waves and to allow a compensational outflow of perilymphatic fluid, the electrode 
insertion is carried out slowly, over a period of approximately 1-2 minutes. During the insertion of the electrode, the residual cochlear function may be monitored via evoked auditory brainstem response (ABR) if a waveform is present. The surgeon must pause during the insertion to allow for the ABR data to be collected. If changes in the ABR are noted, the insertion proceeds at a slower rate.

The electrode is advanced to the fascia "washer" to ensure a tight seal, and the tegmen mastoideum suture is secured. Sealing of the cochleostomy site and the electrode fixation also can be achieved with injection of fibrin glue (Beriplast ${ }^{\circledR}$ ). Because of the desire to preserve residual hearing, the middle ear is not packed with muscle or fascia. The mastoid periostium should then be closed completely over the receiver/stimulator and the electrode. The skin and soft tissues are then closed in the standard fashion.

\subsection{Cochleostomy versus round window insertion}

During the early years (prior to Lehnhardt et al.'s description of a soft surgery technique in 1993) of cochlear implantation emphasis was on implanting a safe and reliable electrode array into the bony cochlear channel. Entrance was obtained via the round window (as the reliable landmark) but relatively stiff multichannel electrode designs and the hook region of the cochlea led to difficult insertions and injury to the osseous spiral lamina. This bony structure transmits the peripheral auditory nerve fibers. Damage to this delicate bone could then negatively impact a patient's ability to perceive sound provided by the CI. As a result, the round window technique originally used in cochlear implantation was abandoned because of concerns that the angle of insertion lead to trauma of the osseous spiral lamina. It was then proposed that by drilling the promontory bone, one could reliably gain access to the intracochlear lumen. This procedure has been generally known as the cochleostomy. The classic cochleostomy approach has become popular among most otologists as it provides reliable access to the scale tympani of the cochlea.

Briggs et al. (2005) studied 27 temporal bones and described the complex anatomy of the hook region of the cochlea, especially in relation to the optimal placement of the cochleostomy for electrode insertion during hearing preservation surgery. They recommended that the cochleostomy be performed directly inferior to the round window membrane through the crista fenestra (the ridge of bone immediately inferior of the round window membrane) to minimize intracochlear damage during electrode insertion.

However, with the creation of softer, more flexible electrode arrays some authors have revisited the concept of a round window insertion technique. Adunka et al. (2004) described a method of cochlear implantation using the round window membrane insertion technique in eight human fresh temporal bones. Using the standard Combi $40+$ and the FLEXEAS electrode, manufactured by Med-El, they implanted these electrodes through the round window membrane. Using the electrode insertion trauma (EIT) grading system (Table 1), they found that the round window insertion was less traumatic, especially in the basal parts of the cochlea, compared with the cochleostomy approach.

There are proponents of both approaches (Roland et al., 2008; Adunka et al., 2004; Gantz et al., 2005; Skarzynski et al., 2007; Wright \& Roland, 2005). However, it should be noted that the angle of insertion for round window electrode insertion is not anatomically 
straightforward and should only be considered for patients who have laterally facing round window membrane.

A bony cochleostomy is appropriate for most patients and can be fashioned to receive the different commercially available electrodes. The Cochlear Corporation Hybrid S12 and L24 are designed to be inserted through a bony cochleostomy. The Med-El FLEXEAS can be inserted through either a cochleostomy or a round window insertion.

\section{Post-implantation hearing loss: Potential causes \& therapy}

Despite advances in both surgical techniques and new less traumatic electrode designs some patients still lose some or all of their residual hearing after implantation.

A recent study in rats characterized the hearing loss following EIT. Eshrahgi et al. (2005) reported an initial hearing loss of 25 to $35 \mathrm{~dB}$ sound pressure level (SPL) and then a progressive loss of hearing of an additional $15 \mathrm{~dB}$ SPL postimplantation (i.e.; Days 0-7) for all of the frequencies tested (i.e.; 4-32 kHz). Subsequently these investigators confirmed the same pattern of hearing loss due to EIT in a guinea pig model (Eshraghi et al., 2006). The cochleae of control guinea pigs and implanted guinea pigs were removed at 12, 24, and 36 hours after surgery for analysis. Interestingly, they observed changes in nuclear morphology (i.e. nuclear condensation and fragmentation) of the sensory hair cells of the traumatized cochlea at a site distal to the initial site of EIT that are consistent with apoptosis. Furthermore, there was a progressive increase in terminal deoxynucleotide transferasemediated dUTP nick-end labeling (TUNEL)-labeled hair cell nuclei in the traumatized cochlea over time compared with the contralateral control cochlea, consistent with apoptotic cell death in hair cells remote from the electrode insertion site.

Many additional animal studies confirmed that the insertion of a cochlear implant electrode array causes cellular injury on a molecular level that cannot be correlated to the degree of direct physical trauma. With these observations, grade 0 physical trauma is now considered as no observable macroscopic damage, but with the possibility of damage on a molecular level that results in EIT-induced hearing loss (Table 1).

\subsection{Mechanism of cell death}

Auditory hair cells may die via necrosis, necrosis-like programmed cell death (PCD), apoptosis, or any combination of these mechanisms in response to a variety of insults (eshraghi et al., 2006; Do et al., 2004). The insertion of a cochlear implant electrode array into the scala tympani can directly kill residual hair cells via necrosis. It can also lead to oxidative stress within the damaged tissues of the cochlea resulting in the apoptosis of hair cells and subsequent loss of hearing. Necrosis is a passive consequence of an overwhelming injury to a cell and is marked by nuclear swelling and lysis of the affected cell. The lysis of the cell results in exposure of the intracellular contents to the extracellular environment and provokes an inflammatory response. The inflammatory response itself may cause further localized tissue destruction as inflammatory cells are recruited to the injured site.

In contrast to necrotic cell death, apoptosis (type 1 PCD) and necrosis-like programmed cell death (type 2 PCD) are associated with an active cell death process and involve a series of biochemical intracellular signaling events that occur in response to injury (Fig. 5). After an 
insult, PCD protects the organism by removing cells that have sustained sufficient damage to become potentially harmful to the integrity of a tissue or organ. The cytoplasm and nuclear chromatin of the injured cell condense; ribosomes and mitochondria aggregate; and the cell begins to die by forming cellular fragments called apoptotic bodies. While there is blebbing in the plasma membrane, its integrity is largely retained so that, unlike necrosis, and an inflammatory response is not initiated. The biochemical cascade within an apoptotic cell involves the orderly fragmentation of DNA. These fragments, called nick-ends, offer multiple sites for labeling with chemicals during preparation of tissue for examination by microscopy. For example, TUNEL allows for the objective identification of cells dying by apoptosis.

Apoptosis is a tightly controlled process within the cell. Members of the Bcl-2 family of pro (e.g., Bax and Bid) and antiapoptotic (e.g., Bcl-2 and Bcl- $\mathrm{X}_{\mathrm{L}}$ ) proteins, cytochrome c and some of the members of the family of caspase proteases all regulate entry into PCD (Hertz et

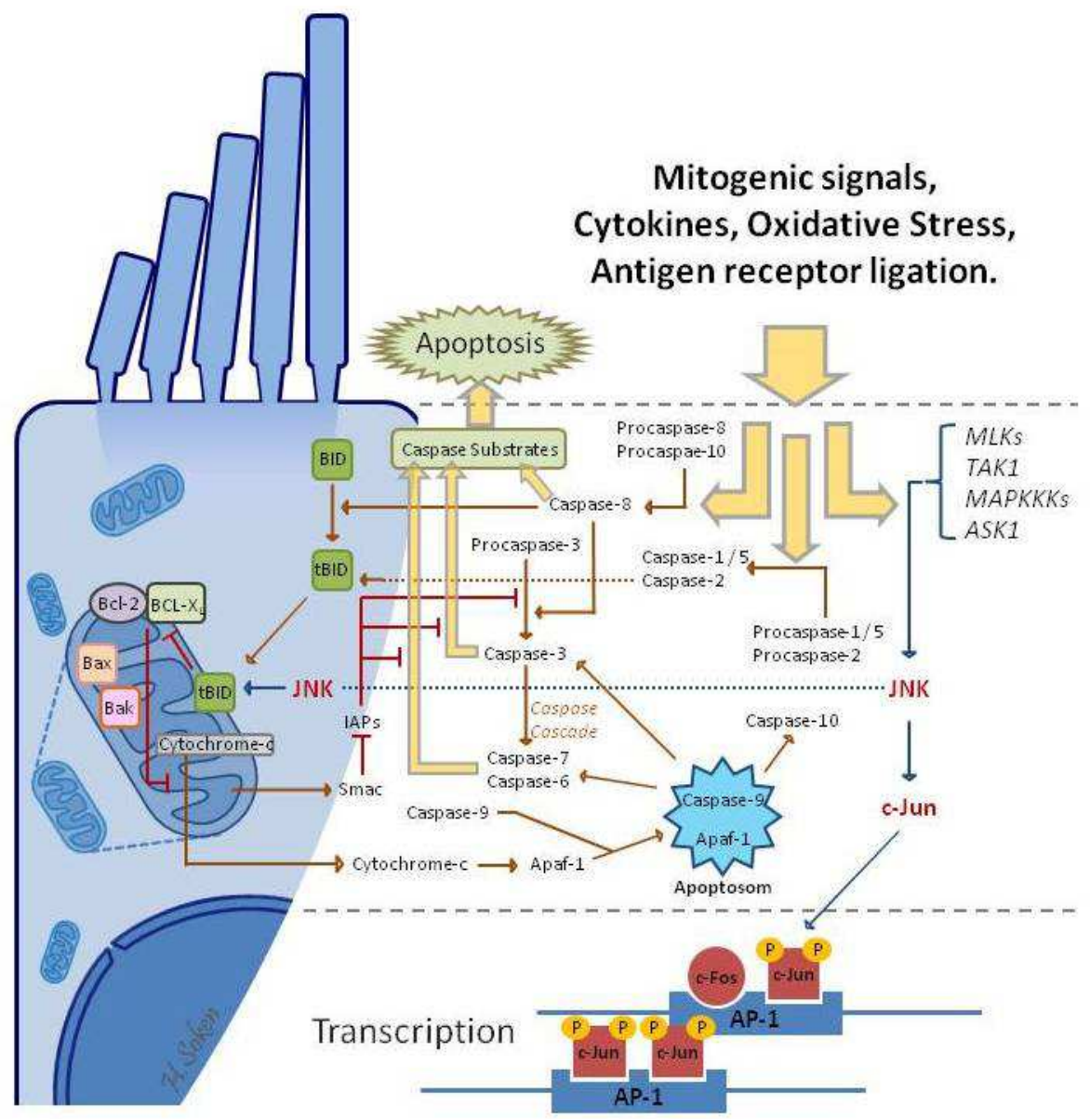

Fig. 5. Overview of caspase pathway and signal transduction cascades involved in apoptosis. 
al., 2005; Kim et al., 2005). Caspases are a family of aspartate-specific cysteine proteases, which exist as latent intracellular zymogens (Van De Water et al., 2004). Effector caspases, once activated, selectively cleave distinct intracellular substrates that lead to the dismantling of a cell's architecture, DNA, signaling apparatus, and restorative repair mechanisms. The sequence of caspase activation shows that distinct cascades are activated depending on the specific pathology, conditions employed, and the cell type. At present, caspases 8, 9, and 3 are known to be involved in the apoptosis of physically damaged hair cells (Nicotera et al., 2003), caspases 5, 6, 7 and 10 are suggested to participate in the apoptosis of hair cells in response to physical trauma (Do et al., 2004; Van De Water et al., 2004) (Fig. 5). The underlying events include loss of mitochondrial transmembrane potential, release of cytochrome $\mathrm{c}$ from the damaged mitochondria into the cytoplasm, formation of an apoptosome, sequential activation of activator and then effector procaspases, and a subsequent increase in lipid peroxidation of cellular membranes.

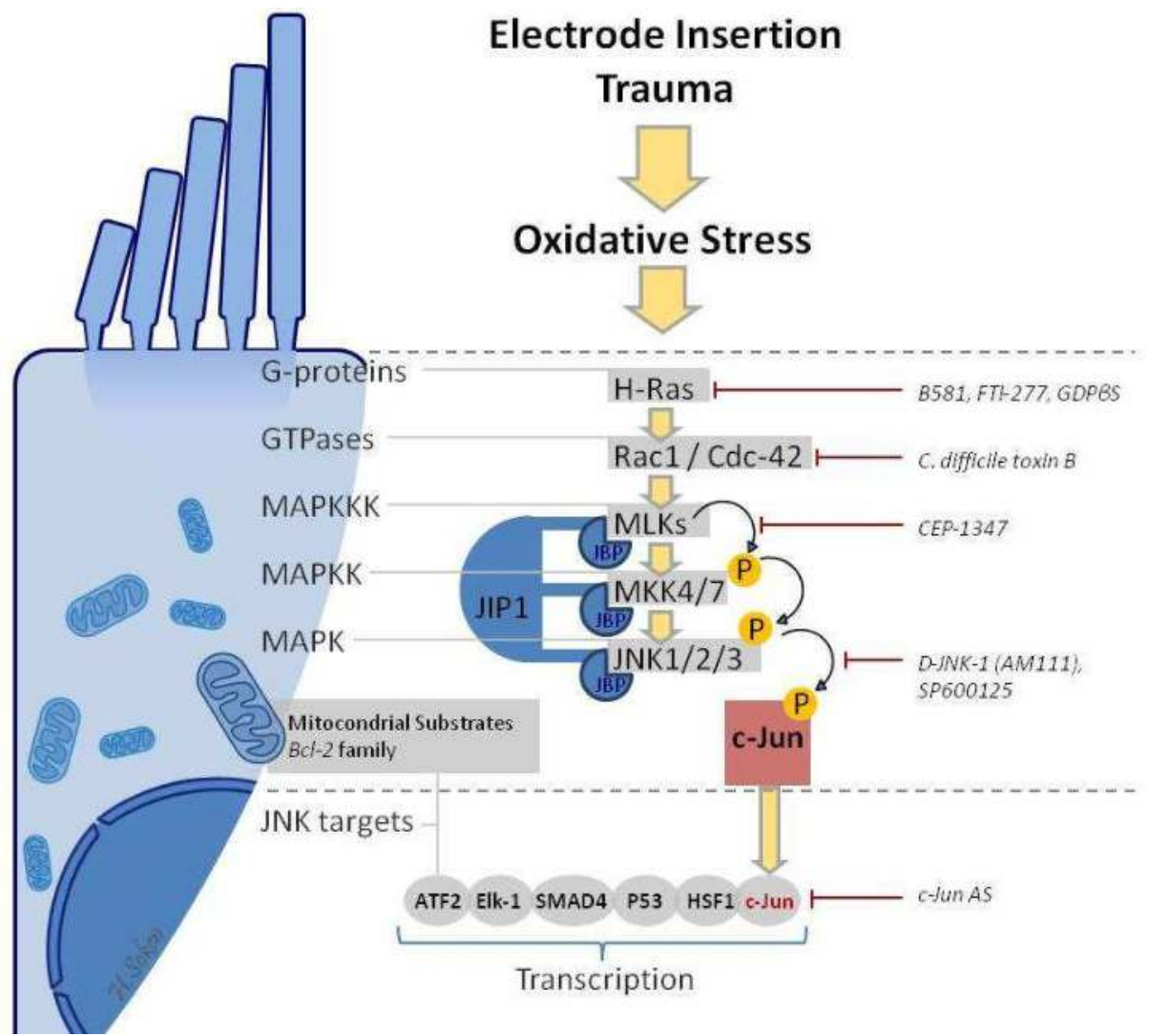

Fig. 6. The MAPK/JNK cell death signal cascade and the site of activation of many pharmacologic inhibitors that have been demonstrated either to block or partially to block different points within this signaling pathway. The mechanism of activation by which DJNK-1 blocks the actions of JNK, with c-Jun being used as the example of a major downstream target. 
Another proposed molecular pathway for cellular insult to apoptosis is via the activation and expression of immediate early genes, such as c-jun (Atkins et al., 1996). Jun:Jun homodimers and Jun:Fos heterodimers are major components of the activator protein-1 (AP1) transcription complex (Fig. 6). Strong c-Jun/AP-1 activation is found in apoptotic cells during the process of naturally occurring (programmed) cell death in the developing rat brain (Ferrer et al., 1996). The phosphorylation cascade necessary for c-Jun activation has also been established. In response to a variety of cell injuries, c-Jun N-terminal kinase (JNK) phosphorylates c-Jun, stimulating its ability to activate transcription of target genes. JNK is a member of the MAP kinase family and is itself regulated by a phosphorylation via a kinase cascade (Kyriakis \& Avruch, 1996; Derijard et al., 1995) (Fig. 6). This JNK/c-Jun cell death pathway contributes to the loss of hair cells and auditory neurons in response to oxidative stress (Pirvola et al., 2000; Scarpidis et al., 2003; Wang et al., 2003).

\subsection{Pharmaceutical approaches and drug treatment}

To maximize hearing preservation after electrode insertion and to enhance the performance of the cochlear implant, direct delivery of pharmacological agents to the inner ear is under active investigation. The invasive nature of cochlear implant electrode insertion itself provides both an opportunity for direct local drug delivery and a platform for the development of a delivery device, e.g., syringes, osmotic pumps, cochlear prosthesis-based delivery and other newer devices have been employed.

\subsection{1 c-Jun N-terminal Kinase (JNK) inhibitors}

To prevent apoptosis of injured auditory hair cells, one strategy involves the use of a highly effective peptide JNK inhibitor (D-JNKI-1). D-JNKI-1 (also known as AM 111) is a cellpermeable peptide which acts by interrupting the MAPK/JNK signal cascade at the level of the three JNK molecular isoforms. This inhibition prevents the phosphorylation of c-Jun, and thus disrupts the formation of an AP-1 transcription factor. D-JNK-1 also prevents the JNKs from disrupting the activity of antiapoptotic members of the Bcl-2 family and inhibits activation of other JNK targets (e.g., ATF-2) (Figs. 5, 6).

A previous study using D-JNK-1 has demonstrated that this inhibitory peptide can prevent loss of both hearing capacity and hair cells in animals challenged with exposure to either a damaging level of sound trauma or to an ototoxic level of aminoglycoside antibiotic (Wang et al., 2003). Using guinea pig models of cochlear implant trauma Van de Water, et al., showed that treatment with D-JNK-1 prevented the progressive increase in ABR thresholds and decrease in DPOAE amplitudes that occur after electrode insertion trauma (Eshraghi et al., 2006). In another study, Barkdull et al. (2007) confirmed the efficacy of D-JNKI-1 in preventing hearing loss caused by inflammation.

D-JNKI-1, represent promising potential therapeutics for the prevention of hearing loss during electrode insertion in partial hearing patients. In sum, the delayed progressive component of EIT- hearing loss may be mitigated by treating the cochlea immediately after insertion with a JNK inhibitor. Further research in this area is ongoing.

\subsubsection{Caspase inhibitor therapy}

Among antiapoptotic agents, the general caspase inhibitor z-VAD-FMK (carbobenzoxyvalyl-alanyl-aspartyl-[O-methyl]-fluoromethylketone) is also currently under investigation. 
Z-VAD-FMK is a cell-permanent pan-caspase inhibitor that irreversibly binds to the catalytic site of caspase proteases thus inhibiting apoptosis. To be effective, Z-VAD-FMK should be added at the same time that apoptosis is induced (Wang et al., 2004).

Animal experiments have shown caspase inhibition by Z-VAD-FMK can rescue hair cells from the lethal effects of aminoglycosides or cis-platinum (Wang et al., 2004; Matsui et al., 2003). Do et al. (2004) found in their mouse model of implantation trauma that the use of cell death inhibitors (e.g., pancaspase inhibitor Z-VAD-FMK and specific inhibitors to caspase 3, 5, and 6) significantly protected the hearing in response to hydraulic trauma.

\subsubsection{Mild hypothermia therapy}

Necrosis is generally thought to occur rapidly and therefore is thought to be very difficult to reverse with most otoprotection treatments, e.g. z-VAD-fmk or JNK inhibitors. Reasonable approaches to the prevention of trauma induced necrosis of hair cells would be to either prevent the insult from occurring (e.g. modified surgical approach) (Eshraghi et al., 2003) or to slow the metabolic response of a cell to injury as can be accomplished by the application of protective hypothermia (Balkany et al., 2005).

Hypothermia has been shown to have a protective effect in the brain following a traumatic injury (Busto et al., 1987; Dietrich et al., 1994; Kil et al., 1996). The beneficial effect of hypothermia on neuronal injury has been attributed to a variety of mechanisms. These include a reduction in metabolic rate, reduced tissue oxygen consumption, decreased metabolic acidosis, a suppression of calcium influx into neurons, diminished nitric oxide production, and a reduction in the level of glutamate excitotoxicity (Hyodo et al., 2001).

Hypothermia has also been demonstrated to reduce brain damage following ischemia by limiting the extent of oxidative stress (Zhao et al., 1996). More recently, it was reported that hypothermia could protect against noise-induced hearing threshold elevation in mice (Henry, 2003). In a recent study, it has been shown that mild hypothermia can reduce the immediate component of trauma-induced hearing loss and prevent the progressive component of loss of auditory function following cochlear electrode insertion in the rat model of cochlear implantation trauma-induced hearing loss (Balkany et al., 2005).

\subsubsection{Glucocorticoid therapy}

Glucocorticoid receptors are expressed in the inner ear. In the adult rat cochlea, they are expressed in the stria vascularis, the organ of Corti and the spiral ganglion neurons. At the cellular level glucocorticoids provide a wide spectrum of cytoprotective activities, including antioxidant and homeostatic effects. In vitro experiments have shown that steroids have a protective effect on cultured hair cells. Further, tumor necrosis factor $a$, an important inflammatory mediator, is released after injury of the cochlea and induces the loss of auditory hair cells; an effect which is inhibited by dexamethasone. Experiments performed on guinea pigs showed that local treatment of the cochlea with dexamethasone reduces EITinduced hearing loss (James et al., 2008).

A number of groups have investigated the use of steroids using a variety of delivery methodologies (e.g., elution from the electrode carrier, intracochlear injection prior to electrode insertion, delivery through a gel-filled reservoir in the electrode carrier], 
demonstrating efficacy in the protection of hearing after mild to moderate levels of trauma (James et al., 2008; Eshraghi et al., 2007; Ye et al., 2007; Vivero et al., 2008). Thus, glucocorticoids represent another class of potential therapeutical compounds to mitigate hearing loss following cochlear implantation.

\section{Conclusion}

Preservation of residual hearing provides significant audiologic advantages and enhances overall performance in patients receiving cochlear implants; preservation of residual hearing should be a goal of all future CI surgeries. This requires intimate knowledge of cochlear microanatomy and careful microsurgical techniques to limit intracochlear damage. In addition, emerging otoprotective therapeutic strategies may help maintain residual auditory function after implantation. Taken together, these microsurgical and therapeutic strategies should lead to improved outcomes for cochlear implant recipients.

\section{References}

Adunka O, Unkelbach MH, Mack M, et al. (2004). Cochlear implantation via the round window membrane minimizes trauma to cochlear structures: a histologically controlled insertion study. Acta Otolaryngol. Vol.124, No.7, pp. 807-812

Atkins PT, Liu PK, Hsu CY. (1996). Immediate early gene expression in response to cerebral ischemia, friend or foe? Stroke. Vol.27, pp. 1682-7

Balkany T, Eshraghi AA, Yang N. (2002). Modiolar proximity of three new perimodiolar cochlear implant electrodes. Acta Otolaryngol. Vol.122, pp. 363-369

Balkany TJ, Connell SS, Hodges AV, et al. (2006). Conservation of residual acoustic hearing after cochlear implantation. Otol Neurotol. Vol.27, No.8, pp. 1083-8

Balkany TJ, Eshraghi AA, He J, Polak M, Mou C, Dietrich WD, Van De Water TR. (2005). Mild hypothermia protects auditory function during cochlear implant surgery. Laryngoscope. Vol.115, pp. 1543-1547

Barkdull GC, Hondarrague Y, Meyer T, Harris JP, Keithley EM. (2007). AM-111 reduces hearing loss in a guinea pig model of acute labyrinthitis. Laryngoscope. Vol.117, No.12, pp. 2174-2182

Briggs R, Tykocinski M, Stidham K, Roberson JB. (2005). Cochleostomy site: implication for electrode placement and hearing preservation. Acta Otolaryngol. Vol.125, No.8, pp. 870-876

Busto R, Dietrich WD, Globus MY-T, et al. (1987). Small differences in intra-ischemic brain temperature critically determine the extent of ischemic neuronal injury. J Cereb Blood Flow Metab. Vol.7, pp. 729-738.

Ching T, Dillon H, Byrne D. (1998). Speech recognition of hearing-impaired listeners: Predictions from audibility and the limited role of high-frequency amplification. Journal of the Acoustical Society of America. Vol.103, No.2, pp. 1128-1140

Cohen NL, Roland JT, Fishman A. (2002). Surgical technique for the Nucleus Contour cochlear implant. Ear Hear. Vol.23, pp. 59-66

Derijard B, Raingeand J, Barrett T, et al. (1995). Independent human MAP-kinase signal transduction pathways defined by MEK and MKK isoforms. Science. Vol.267, pp. $682-5$ 
Dietrich WD, Alonso O, Busto R, et al. (1994). Post-traumatic brain hypothermia reduces histopathological damage following concussive brain injury in the rat. Acta Neuropathol. Vol.87, pp. 250-258

Do K, Baker K, Praetorius M, Staecker H. (2004). A mouse model of implantation trauma. Internat Cong Ser. Vol.1273, pp. 167-170

Eshraghi AA, Polak M, He J, et al. (2005). The pattern of hearing loss in a rat model of cochlear implantation trauma. Otol Neurotol. Vol.26, pp. 442-447

Eshraghi AA, Van De Water TR. (2006). Cochlear function, physical trauma, oxidative stress, induction of apoptosis and therapeutic strategies. Anat Rec. Vol.288A, pp. 473-481

Eshraghi AA, Wang J, Adil E, He J, Zine A, Bublik M, Bonny C, Puel JL, Balkany TJ, Van De Water TR. (2007). Blocking c-Jun-Nterminal kinase signaling can prevent hearing loss induced by both electrode insertion trauma and neomycin ototoxicity. Hear Res. Vol.226, pp. 168-177

Eshraghi AA, YangNW, Balkany TJ. (2003). Comparative study of cochlear damage with three perimodiolar electrode designs. Laryngoscope. Vol.113, pp. 415-419

Fayad J, Linthicum FR Jr, Otto SR, et al. (1996). Cochlear implants: histopathologic findings related to performance in 16 human temporal bones. Ann Otol Rhinol Laryngol. Vol.100, pp. 807-811

Ferrer I, Segu1 J, Olive M. (1996). Strong c-Jun immunoreactivity is associated with apoptotic cell death in human tumors of the central nervous system. Neurosci Lett. Vol.214, pp. $49-52$

Gantz BJ, Hansen MR, Turner CW, et al. (2009). Hybrid 10 Clinical trial. Audiol Neurotol. Vol.14(suppl 1), pp. 32-38

Gantz BJ, Turner CW, Gfeller KE, et al. (2005). Preservation of hearing in cochlear implant surgery: Advantages of combined electrical and acoustic speech processing. Laryngoscope. Vol.115, No.5, pp. 796-802

Gantz BJ, Turner CW, Gfeller KE. (2006). Acoustic plus electric speech processing: preliminary results of a multicenter clinical trial of the Iowa/Nucleus Hybrid implant. Audiol Neurootol. Vol.11(Suppl 1), pp. 63-68

Gantz BJ, Turner CW. (2003). Combining acoustic and electric hearing. Laryngoscope. Vol.113, pp. 1726-1730

Gfeller KE, Olszewski C, Turner CW, et al. (2006). Music perception with cochlear implants and residual hearing. Audiol Neurotol. Vol.11(suppl 1), pp. 12-15

Gfeller KE, Turner CW, Oleson J, et al. (2007). Accuracy of cochlear implant recipients on pitch perception, melody recognition, and speech reception in noise. Ear Hear. Vol.28, No.3, pp. 412-423

Gstoettner WK, Helbig S, Maier N, et al. (2006). Ipsilateral electric acoustic stimulation of the auditory system: results of long-term hearing preservation. Audiol Neurotol. Vol.11(Suppl 1), pp. 49-56

Gstoettner WK, Helbig S, Maier N, Kiefer J, Radeloff A, Adunka OF. (2006). Ipsilateral electric acoustic stimulation of the auditory system: results of long-term hearing preservation. Audiol Neurootol. Vol.11(Suppl 1), pp. 49-56

Gstoettner WK, van de Heyning P, O'Connor AF, et al. (2008). Electric acoustic stimulation of the auditory system: results of a multi-centre investigation. Acta Otolaryngol. Vol.128, No.9, pp. 968-975 
Henry KR. (2003). Hyperthermia exacerbates and hypothermia protects from noise-induced threshold elevation of the cochlear nerve envelope response in C57BL/J mouse. Hear Res. Vol.179, pp. 88-96

Hertz CA, Torres V, Quest AFG. (2005). Beyond apoptosis: non-apoptotic cell death in physiology and disease. Biochem Cell Biol. Vol.83, pp. 579-588

Hochmair I, Nopp P, Jolly C, et al. (2006). Med-El cochlear implants: State of the art and a glimpse into the future. Trends in Amplification. Vol.10, No.4, pp. 201-220

Hodges AV, Schloffman J, Balkany T. (1997). Conservation of residual hearing with cochlear implantation. Am J Otol. Vol.18, No.2, pp. 179-183

Hogan C, Turner CW. (1998). High-frequency amplification: benefits for hearing-impaired listeners. Journal of the Acoustical Society of America. Vol.104, No.1, pp. 432-441

Hyodo J, Hakuba N, Koga K, Watanabe F, Shudou M, Taniguchi M, Gyo K. (2001). Hypothermia reduces glutamate efflux in perilymph following transient cochlear ischemia. Neuroreport. Vol.12, pp. 1983-1987

James DP, Eastwood H, Richardson RT, O'Leary SJ. (2008). Effects of round window dexamethasone on residual hearing in guinea pig mode; of cochlear implantation. Audiol Ear Institute. Vol.13, pp. 86-96

Kiefer J, von Ilberg C, Reimer B, et al. (1998). Results of cochlear implantation in patients with severe to profound hearing loss-implications for the indications. Audiology. Vol.37, pp. 382-95

Kil HY, Zhang J, Piantadosi C. (1996). Brain temperature alters hydroxyl radical production during cerebral ischemia/reperfusion in rats. J Cereb Blood Flow Metab. Vol.16, pp. 100-106

Kim R, Emi M, Tanabe K. (2005). Role of mitochondria as the gardens of cell death. Cancer Chemother Pharmacol. Vol.21, pp. 1-9

Klenzner T, Stecker M, Marangos N, Laszig R. (1999). Extended indications for cochlear implantation. The Freiburg results in patients with residual hearing. HNO. Vol.47, pp. 95-100

Kyriakis JM, Avruch J. (1996). Sounding the alarm: protein kinase cascades activated by stress and inflammation. J Biol Chem. Vol.271, pp. 24313-6

Lehnhardt E. (1993). Intracochlear placement of cochlear implant electrode in soft surgery technique. HNO. Vol.41, pp. 356-359.

Lenarz TA, Stover T, Buechner A, et al. (2009). Hearing conservation surgery using the Hybrid-L electrode. Audiol Neurotol. Vol.14(suppl 1), pp. 22-31

Matsui JI, Haque A, Huss D, Messana EP, Alosi JA, Roberson DW, Cotanche DA, Dickman JD, Warchol ME. (2003). Caspase inhibitors promote vestibular hair cell survival and function after aminoglycoside treatment invivo. J Neurosci. Vol.23, pp. 61116122

Nelson P, Jin SH, Carney AE, et al. (2003). Understanding speech in modulated interference: cochlear implant uses and normal-hearing listeners. J Acoust Soc Am. Vol.113, No.2, pp. 961-968

Ni D, Shepherd RK, Seldon HL, et al. (1992). Cochlear pathology following chronic electrical stimulation of the auditory nerve. I: Normal hearing kittens. Hear Res. Vol.62, No.1, pp. 63-81

Nicotera TM, Hu BH, Hendrson D. (2003). The caspase pathway in noise-induced apoptosis of the chinchilla cochlea. J Assoc Res Otolaryngol. Vol.4, pp. 466-477 
Pirvola, U., Xing-Qun, L., Virkkala, J., Saarma, M., Murakata, C., Camoratto, A.M., Walton, K.M., Ylikoski, J. (2000). Rescue of hearing, auditory hair cells, and neurons by CEP-1347/KT7515, an inhibitor of c-Jun N-terminal kinase activation. J. Neurosci. Vol.20, pp. 43-50

Reiss LA, Turner CW, Erenberg SR, et al. (2007). Changes in pitch with a cochlear implant over time. JARO. Vol.8, pp. 241-257

Roland JT Jr, Zeitler DM, Jethanamest D, et al. (2008). Evaluation of the short hybrid electrode in human temporal bones. Otol Neurotol. Vol.29, No.4, pp. 482-488

Scarpidis BC, Madnani D, Shoemaker C, et al. (2003). Arrest of apoptosis in auditory neurons: implications for sensorineural preservation in cochlear implantation. Otol Neurotol. Vol.24, pp. 409-417

Skarzynski H, Lorens A, Piotrowska A, et al. (2007). Preservation of low frequency hearing in partial deafness cochlear implantation (PDCI) using the round window surgical approach. Acta Otolaryngol. Vol.127, No.1, pp. 41-48

Turner CW, Cummings KJ. (1999). Speech audibility for high-frequency hearing loss listeners. American Journal of Audiology. Vol.8, pp. 47-56

Turner CW, Gantz BJ, Vidal C, et al. (2004). Speech recognition in noise for cochlear implant listeners: benefits of residual acoustic hearing. J Acoust Soc Am. Vol.115, No.4, pp. 1729-1735

Van De Water TR, Lallemend F, Eshraghi AA, et al. (2004). Caspases, the enemy within, and their role in oxidative stress-induced apoptosis of the inner ear sensory cells. Otol Neurotol. Vol.25, pp. 627-632

Vivero RJ, Joseph DE, Angeli S, He J, Chen S, Eshraghi AA, Balkany TJ, Van de Water TR. (2008). Dexamethasone base conserves hearing from electrode trauma induced hearing loss. Laryngoscope. Vol.118, pp. 2028-2033

von Ilberg C, Kiefer J, Tillein J, et al. (1999). Electric-Acoustic stimulation of the auditory system. New Technology for severe hearing loss. ORL J Otorhinolaryngol Relat Spec. Vol.61, No.6, pp. 334-40

Wang J, Ladrech S, Pujol R, Brabet P, Van De Water TR, Puel JL. (2004). Caspase inhibitors, but not c-Jun NH2-terminal kinase inhibitor treatment, prevent cisplatin induced hearing loss. Cancer Res. Vol.64, pp. 9217-9224

Wang J, Van De Water TR, Bonny C, et al. (2003). A peptide inhibitor of c-Jun N-Terminal Kinase (D-JNKI-1) protects against both aminoglycoside and acoustic traumainduced auditory hair cell death and hearing loss. J Neurosci. Vol.23, pp. 596-8607

Wright CG, Roland PS. (2005). Temporal bone microdissection for anatomic study of cochlear implant electrodes. Cochlear Implants Int. Vol.6, No.4, pp. 159-168

Xu J, Shepherd RK, Millard RE, et al. (1997). Chronic electrical stimulation of the auditory nerve at high stimulus rates: a physiological and histopathological study. Hear Res. Vol.10(1-2), pp. 1-29

Yao, A., Turner, C.W., Gantz, B.J. (2006). Stability of low-frequency residual hearing in patients who are candidates for combined acoustic plus electric hearing. J. Speech Hear. Res. Vol.49, pp. 1085-1090

Ye Q, Tillein J, Hartmann R, Gstoettner W, Kiefer J. (2007). Application of a corticosteroid (Triamcinolon) protects inner ear function after surgical intervention. Ear Hear. Vol.28, pp. 361-369 
Zhao W, Richardson JS, Mombourquette MJ, Weil JA, Ljaz S, Shuaib A. (1996). Neuroprotective effects of hypothermia and U-78517F in cerebral ischemia are due to reducing oxygen-based free radicals: an electron paramagnetic resonance study with gerbils. J Neurosci Res. Vol.45, pp. 282-288 


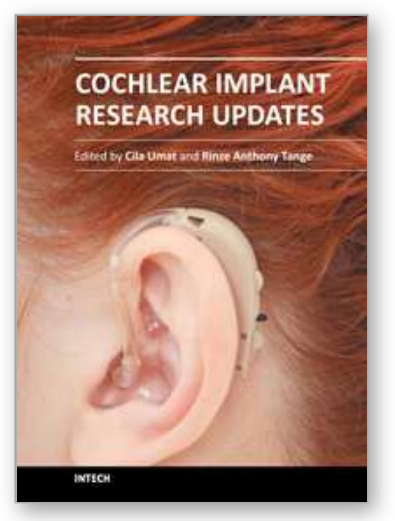

\author{
Cochlear Implant Research Updates \\ Edited by Dr. Cila Umat
}

ISBN 978-953-51-0582-4

Hard cover, 232 pages

Publisher InTech

Published online 27, April, 2012

Published in print edition April, 2012

For many years or decades, cochlear implants have been an exciting research area covering multiple disciplines which include surgery, engineering, audiology, speech language pathology, education and psychology, among others. Through these research studies, we have started to learn or have better understanding on various aspects of cochlear implant surgery and what follows after the surgery, the implant technology and other related aspects of cochlear implantation. Some are much better than the others but nevertheless, many are yet to be learnt. This book is intended to fill up some gaps in cochlear implant research studies. The compilation of the studies cover a fairly wide range of topics including surgical issues, some basic auditory research, and work to improve the speech or sound processing strategies, some ethical issues in language development and cochlear implantation in cases with auditory neuropathy spectrum disorder. The book is meant for postgraduate students, researchers and clinicians in the field to get some updates in their respective areas.

\title{
How to reference
}

In order to correctly reference this scholarly work, feel free to copy and paste the following:

Hakan Soken, Sarah E. Mowry and Marlan R. Hansen (2012). Cochlear Implant Surgery, Cochlear Implant Research Updates, Dr. Cila Umat (Ed.), ISBN: 978-953-51-0582-4, InTech, Available from: http://www.intechopen.com/books/cochlear-implant-research-updates/hearing-preservation-in-cochlearimplant-surgery

\section{INTECH}

open science | open minds

\section{InTech Europe}

University Campus STeP Ri

Slavka Krautzeka 83/A

51000 Rijeka, Croatia

Phone: +385 (51) 770447

Fax: +385 (51) 686166

www.intechopen.com

\section{InTech China}

Unit 405, Office Block, Hotel Equatorial Shanghai

No.65, Yan An Road (West), Shanghai, 200040, China

中国上海市延安西路65号上海国际贵都大饭店办公楼 405 单元

Phone: +86-21-62489820

Fax: $+86-21-62489821$ 
(C) 2012 The Author(s). Licensee IntechOpen. This is an open access article distributed under the terms of the Creative Commons Attribution 3.0 License, which permits unrestricted use, distribution, and reproduction in any medium, provided the original work is properly cited. 